revealed that Lysinibacillus sphaericus significantly reduced the aspirin levels in aspirin-containing medium. Moreover, the germ-free mice monocolonized with Lysinibacillus sphaericus showed significantly lower plasma aspirin levels post aspirin administration relative to the intact germ-free mice, confirming a role of this bacterium in reducing aspirin bioavailability.

Conclusions Gut microbiota modulates the chemopreventive efficacy of aspirin on CRC through impact on aspirin bioavailability in mice. Lysinibacillus sphaericus may play a critical role in aspirin degradation in the gut.

\section{IDDF2019-ABS-0200 LONG NON-CODING RNA LOC148709 REGULATES PFKFB3-MEDIATED GLYCOLYTIC REPROGRAMMING IN ESOPHAGEAL SQUAMOUS CELL CARCINOMA}

${ }^{1}$ Jia Liu*, ${ }^{1}$ Ze-Xian Liu, ${ }^{1}$ Qi-Nian Wu, ${ }^{1}$ Yun-Xin Lu, ${ }^{2}$ Chau-Wei Wong, ${ }^{1}$ Huai-Qiang Ju, ${ }^{1}$ RuiHua Xu. ${ }^{1}$ Sun Yat-sen University Cancer Center, China; ${ }^{2}$ The First Affiliated Hospital of Sun Yat-sen University, China

10.1136/gutjnl-2019-IDDFabstracts.37

Background Rapid proliferation and glucose metabolism remodeling are hallmarks of cancers. Long non-coding RNAs (lncRNAs) are potentially involved in Warburg effect. We aimed to identify oncogenic lncRNAs that significantly affect the development of esophageal squamous cell carcinoma (ESCC) and investigate the metabolism-related mechanisms.

Methods Bioinformatics analysis and siRNA library screening were used to pinpoint IncRNAs that significantly affected cell glycolysis and proliferation. RNAScope(R) in situ hybridization and qRT-PCR assays were performed in clinical samples to investigate expression levels and clinical relevance of the lncRNA. RNA interference and CRISPR-Cas9 were used to explore the functional roles of the lncRNA. In vivo, cell-based and patient-derived xenograft (PDX) models were used. Extracellular acidification rate and ${ }^{13} \mathrm{C}$-labeled intracellular metabolites were determined. RNA pull-down, MS2-Tagged RNA affinity purification, RNA-binding protein immunoprecipitation and cross-linking immunoprecipitation were performed to identify lncRNA associated proteins and related mechanisms.

Results The IncRNA LOC148709 was identified as a metabolism-related IncRNA. Increased expression of LOC148709 was observed in ESCC and was correlated with poor prognosis. LOC148709 knockdown significantly decreased cell proliferation and glycolysis. Mechanistically, LOC148709 was directly associated with PFKFB3 and significantly affected PFKFB3 stability. LOC148709 interacted mainly with the C-terminal fragment of PFKFB3 and the T5 (2031-2321) fragment of LOC148709 mediated the interaction with PFKFB3. Through inhibiting K302 ubiquitination, LOC148709 protected PFKFB3 from proteasomal degradation, which subsequently activated glycolytic flux and promoted cell cycle progression by regulating p27 and CDK1. P53 could bind to the LOC148709 promoter and repress its transcription, p53 loss or mutation triggered striking LOC148709 upregulation. Multiple microenvironmental factors, including hypoxia and oncogenic stress, were also involved in the LOC148709 regulatory network via affecting the status of p53. Notably, our patient-derived xenograft (PDX) model studies demonstrated that LOC148709 knockdown dramatically impaired tumor growth.

Conclusions The lncRNA LOC148709 plays an essential role in glycolytic reprogramming by binding to and stabilizing
PFKFB3. This study identified a novel metabolism-related lncRNA and revealed a novel mechanism underlying lncRNAmediated cancer metabolism remodeling. Translational studies further implicated that LOC148709 is a promising biomarker for cancer diagnosis and therapy.

\section{IDDF2019-ABS-0203 SCNN1B FUNCTIONS AS A TUMOR SUPPRESSOR IN COLORECTAL CANCER BY INHIBITING RAS-RAF-MEK-ERK PATHWAY}

${ }^{1}$ Qian Yun*, ${ }^{2}$ Chi Chun Wong, ${ }^{2} J u n$ Yu. 'Department of Gastroenterology, Shenzhen University General Hospital, Shenzhen University Clinical Medical Academy, China; ${ }^{2}$ Department of Medicine and Therapeutics, Chinese University of Hong Kong, Hong Kong

\subsection{6/gutjnl-2019-IDDFabstracts.38}

Background The incidence of colorectal cancer (CRC) is rapidly rising worldwide. Understanding the molecular mechanism underlying CRC pathogenesis is critical to uncover novel drug targets and diagnostics for this disease. Our preliminary work identified SCNN1B as an outlier down-regulated in CRC. SCNN1B mRNA and protein expression were down-regulated in primary CRC and CRC cell lines, suggesting that it might function as a tumor suppressor in CRC.

Methods Clinical significance of SCNN1B in evaluated using tissue microarray $(\mathrm{N}=153)$. Biological function of SCNN1B was investigated in CRC cell lines using cell proliferation, apoptosis, cell cycle, cell migration and subcutaneous xenograft assays. Molecular mechanism of SCNN1B was evaluated by Gene Set Enrichment Analysis (GSEA), and assessment of RasRaf-MEK-ERK pathways.

Results In a pilot tissue microarray cohort of CRC $(\mathrm{N}=153)$, SCNN1B protein expression was an independent prognostic factor predicting favorable patient survival. Ectopic expression of SCNN1B in two CRC cell lines inhibited cell proliferation, induced apoptosis and cell cycle arrest, and suppressed cell migration. Gene Set Enrichment Analysis (GSEA) revealed that SCNN1B expression was associated with KRAS signaling pathway. SCNN1B inhibited p-MEK/p-ERK and SRE luciferase activity, confirming blockade of Ras-Raf-MEK-ERK oncogenic signaling. Mechanistically, SCNN1B suppressed the activation of c-Raf by inducing its inhibitory phosphorylation. Ectopic expression of c-Raf rescued cell proliferation and colony formation in SCNN1B-overexpressed CRC cells, confirming c-Raf as a molecular target of SCNN1B.

Conclusions SCNN1B inhibits colorectal tumorigenesis via inhibition of RAS-MEK-ERK signalling. SCNN1B expression may serve as an independent prognostic biomarker for CRC patients.

\section{IDDF2019-ABS-0204 THE IMPACT OF INTENSIVE PATIENT EDUCATIONAL PROGRAMME ON QUALITY OF BOWEL PREPARATION FOR COLONOSCOPY: A RANDOMISED CONTROL TRIAL}

${ }^{1}$ Sivakami Janahiraman*, 'Tay Chan Yen, 'Lim Wen Ling, 'Lee Jie Min, ${ }^{1}$ Khiew Chun Hoe, ${ }^{2}$ Chew Chun Keat, 'Zakry Yahya Onn, ${ }^{1}$ Irina Ishak, ${ }^{1}$ Mohd Razali Ibrahim. ${ }^{1}$ Hospital Kuala Lumpur, Malaysia; ${ }^{2}$ National Institute of Health, Malaysia

\subsection{6/gutjnl-2019-IDDFabstracts.39}

Background Optimal bowel preparation prior to colonoscopy leads to good quality of visualisation. However, achieving 
high-quality bowel preparation is often difficult. The main aim was to evaluate the impact of the intensive patient educational programme on the quality of bowel preparation. The secondary endpoint was to assess the patient's compliance, acceptability, and tolerability towards the preparation regimen apart from identifying factors associated with good quality bowel preparation.

Methods We performed an assessor-blinded, randomised, controlled trial in an outpatient surgical clinic of a tertiary referral center. Patients were randomly assigned to a control group with standard written and verbal instructions or to an intervention group, where patients received an intensive, structured and comprehensive education programme. The randomisation allocation was concealed in an opaque envelope. All subjects were required to complete a questionnaire as to assess their compliance, acceptability and tolerability towards bowel preparation. Pearson chi-square and multivariable logistics regression were used in statistical analysis.

secondary endpoint

Results Three hundred and twelve patients were randomised, but only 300 patients were included in the analysis. Patients' characteristics of both groups were similar. The proportion of good bowel preparation quality in the interventional group was significantly higher than the control group $(98.7 \%$ versus $52.3 \%, \mathrm{p}<0.001)$. The median total Boston Bowel Preparation Scale (BBPS) score for the interventional group was significantly greater than the control group (8 versus 5, $\mathrm{p}<0.001)$. As compared to the control group, subjects in the interventional group demonstrated higher compliance $(96.6 \%$ vs $85.4 \%, \mathrm{p}<0.001)$, acceptability $(89.3 \%$ vs $33.1 \%, \mathrm{p}<0.001)$ and tolerability $(85.9 \%$ vs $19.2 \%, \mathrm{p}<0.001)$ to bowel preparation regimen. Variables associated to good quality of bowel preparation identified from this study include intensive, structured and comprehensive education programme (OR: 22.5, $\mathrm{p}<0.001,95 \% \mathrm{CI}: 4.05,124.67)$, compliance to bowel cleansing agent (OR: 30.3, p<0.05, 95\% CI: 4.22, 217.01), acceptability of preparation (OR: $2.5, \mathrm{p}<0.05,95 \%$ CI 1.04, 6.05), tolerability towards bowel preparation (OR: 5.3, p $<0.05,95 \%$ CI: 1.55, 18.39) and non-hypomotility drugs (OR: 3.2, $\mathrm{p}<0.05,95 \%$ CI 1.25, 8.43).

Conclusions Patient educational programme is effective in optimizing the quality of bowel preparation for colonoscopy.

\section{IDDF2019-ABS-0209 PAN-TISSUE GENE DYS-REGULATION STUDY OF TYPE II DIABETES BY INTEGRATIVE PATHWAY ANALYSIS}

${ }^{1}$ Xiangtian Yu*, 'Weixin Liu. 'Shanghai Jiao Tong University Affiliated Sixth People's Hospital, Shanghai, China; ${ }^{2}$ Department of Medicine and Therapeutics, The Chinese University of Hong Kong, Hong Kong SAR, China

\subsection{6/gutjnl-2019-IDDFabstracts.40}

Background Different complications and stages often co-occur in the development of Type II Diabetes (T2D). The potential molecular mechanism between complications and related tissues is still not clear, thus it is crucial to characterize the common and specialty of dynamical biological processes in different responsive tissues of Type II Diabetes. Pan-tissue analyses that examine dys-regulation genes and pathways among various tissue types have emerged as a powerful way to obtain novel insights into Type II Diabetes. Considering the definite function modules (e.g. pathways) will be easier to interpret than chaotic genes, we propose a novel computational framework, Tissue Pathway Cross-talk (TPC), to recognize the functional relationship between tissue-specific and tissue-common pathways from omics data .

Methods Here we present TPC investigation on pathway alterations in a pan-tissue cohort including 6 tissues (adipose, liver, Mitochondria, muscle, pancreatic beta-cells, pancreatic islets) from 17 GEO datasets, comprising pathways from KEGG, reactome, Biocarta, and wikiPathway. Using our standardized workflow, we identified betweenness-induced hub-genes from the high-order network connecting common and specific pathways in different tissues.

Results After the comprehensive comparison with the previously reported milestone molecules of $\mathrm{T} 2 \mathrm{D}$, we found:(1) the critical function changes were identified by the tissue-specific pathways, which would be considered as a consensus phenotype-change event during T2D development and progression (table 1); (2) 28 tissue-common pathways related to T2D were significantly enriched with prior-known T2D associated genes, which would be pathway signatures of T2D regulation patterns (table 2); (3) Betweenness-induced hub-genes on pathway-network are selected as the key candidates to study the tissue diversity mechanism under the same disease/T2D condition.

Abstract IDDF2019-ABS-0209 Table 1 Analysis results: The Jaccard Index of pathway intersection between any two tissues.

\begin{tabular}{|c|c|c|c|c|c|c|}
\hline Muscle & $\mathbf{8 5 6}$ & & & & & \\
\hline Liver & $\begin{array}{c}348 \\
(0.342)\end{array}$ & $\mathbf{5 1 0}$ & & & & \\
\hline Mitochondria & $\begin{array}{c}177 \\
(0.186)\end{array}$ & $\begin{array}{c}120 \\
(0.181)\end{array}$ & $\mathbf{2 7 3}$ & & & \\
\hline $\begin{array}{c}\text { Pancreatic beta- } \\
\text { cells }\end{array}$ & $\begin{array}{c}122 \\
(0.132)\end{array}$ & $\begin{array}{c}99 \\
(0.165)\end{array}$ & $\begin{array}{c}44 \\
(0.106)\end{array}$ & $\mathbf{1 8 8}$ & & \\
\hline Adipose & $\begin{array}{c}431 \\
(0.42)\end{array}$ & $\begin{array}{c}282 \\
(0.34)\end{array}$ & $\begin{array}{c}149 \\
(0.20)\end{array}$ & $\begin{array}{c}90 \\
(0.13)\end{array}$ & $\mathbf{6 0 7}$ & \\
\hline Pancreatic islets & $\begin{array}{c}377 \\
(0.365)\end{array}$ & $\begin{array}{c}275 \\
(0.349)\end{array}$ & $\begin{array}{c}103 \\
(0.142)\end{array}$ & $\begin{array}{c}82 \\
(0.124)\end{array}$ & $\begin{array}{c}297 \\
(0.344)\end{array}$ & $\mathbf{5 5 4}$ \\
\hline & Muscle & Liver & Mitochondria & $\begin{array}{c}\text { Pancreatic } \\
\text { beta-cells }\end{array}$ & Adipose & $\begin{array}{c}\text { Pancreatic } \\
\text { islets }\end{array}$ \\
\hline
\end{tabular}

